\section{FATORES MOTIVACIONAIS DE IDOSOS PRATICANTES DO MÉTODO PILATES}

\author{
Motivational factors of elderly practitioners of pilates method \\ Factores de motivación en mayores practicantes del método \\ Pilates
}

\section{RESUMO}

Objetivo: Avaliar os fatores motivacionais de idosos praticantes do Método Pilates e sua relação com o sexo. Métodos: Estudo quantitativo, transversal, realizado no período de abril e maio de 2014, utilizando amostra por conveniência com 39 idosos, independentemente do sexo, praticantes do Método Pilates em um bairro da zona norte do Rio de Janeiro-RJ. Utilizouse o "Inventário de Motivação à Prática Regular de Atividades Físicas (IMPRAF-54)", que avalia as dimensões de motivação ("Controle de Estresse", "Saúde", "Sociabilidade", "Competitividade", "Estética" e "Prazer"). Aplicou-se a estatística descritiva para análise das dimensões. Resultados: Os principais aspectos motivacionais descritos pelos idosos foram "Saúde" (escore bruto médio; mulheres: 34,3( $\pm 4,7)$; homens: 30,3 $( \pm 5,2)$ ), "Sociabilidade" (escore bruto médio; mulheres: 27,8( $\pm 10,2)$; homens: 28,8 $( \pm 9,7))$ e "Prazer" (escore bruto médio; mulheres: $30,8( \pm 7,5)$; homens: $29,3( \pm 8,3))$; somente entre os homens o domínio de "Sociabilidade" foi classificado como "motivação alta" (percentil 70), segundo tabelas normativas do instrumento. Conclusão: Fatores relacionados à saúde, sociabilidade e prazer foram determinantes motivacionais entre os idosos avaliados praticantes do Método Pilates.

Descritores: Motivação; Idoso; Atividade Motora; Exercício.

\section{ABSTRACT}

Objective: To evaluate motivational factors of elderly practitioners of Pilates Method. Methods: Quantitative cross-sectional study conducted from April to May 2014 with a convenience sample of 39 elderly practitioners of Pilates Method - regardless of sexin a neighborhood in the northern part of Rio de Janeiro, RJ. We used the Inventário de Motivação à Prática Regular de Atividades Físicas - IMPRAF-54 (Regular Physical Activity Motivation Inventory), which assesses motivation dimensions ("Stress Control ", "Health", "Sociability", "Competitiveness", "Aesthetics" and "Pleasure"). Results: The main motivational aspects described by the elderly were "Health" (mean crude score; women: 34.3 ( \pm 4.7); men: 30.3 ( \pm 5.2$)$ ), "Sociability" (mean crude score; women: 27.8 ( \pm 10.2$)$; men: 28.8 ( \pm 9.7), and "Pleasure" (mean crude score; women: 30.8 ( \pm 7.5); men: 29.3 ( \pm 8.3$))$; only among men the domain "Sociability" was rated as "high motivation" (70 percentile) according to the normative tables of the instrument. Conclusion: Factors related to health, sociability and pleasure were a key motivation among the elderly practitioners of Pilates Method assessed.

Descriptors: Motivation; Aged; Motor Activity; Exercise.
Artigo Original
1) Universidade Castelo Branco - UCB Rio de Janeiro (RJ) - Brasil

2) Universidade Federal do Rio de Janeiro UFRJ - Rio de Janeiro (RJ) - Brasil

3) Universidade do Estado do Rio De Janeiro - UERJ - Rio de Janeiro (RJ) -

Brasil

Recebido em: 25/05/2015

Revisado em: 30/05/2015 Aceito em: 10/06/2015 


\section{RESUMEN}

Objetivo: Evaluar los factores de motivación en mayores practicantes del Método Pilates y su relación con el sexo. Métodos: Estudio cuantitativo y transversal realizado entre abril y mayo de 2014 con un muestreo por conveniencia de 39 mayores independiente del sexo, practicantes del Método Pilates en un barrio de la zona norte de Río de Janero- RJ. Se utilizó el "Inventario de Motivación para la Práctica Regular de Actividades Físicas (IMPRAF-54)”, que evalúa las dimensiones de motivación ("Control del estrés", "Salud", "Sociabilidad", "Competitividad", "Estética” y "Placer"). Se aplico la estadística descriptiva para el análisis de las dimensiones. Resultados: Los principales aspectos de motivación descritos por los mayores fueron "Salud" (puntuación media bruta; mujeres: 34,3( $\pm 4,7)$; hombres: 30,3( $\pm 5,2)$ ), "Sociabilidad" (puntuación media bruta; mujeres: 27,8( $\pm 10,2)$; hombres: 28,8 ( $\pm 9,7))$ y "Pracer" (puntuación media bruta; mujeres: 30,8( $\pm 7,5)$; hombres: 29,3( $\pm 8,3)$ ); según las tablas normativas del instrumento el dominio "Sociabilidad" fue clasificado como "motivación alta" (percentil 70) solamente entre los hombres. Conclusión: Los factores relacionados a la salud, la sociabilidad y el placer fueron los determinantes de motivación en los mayores practicantes del Método Pilates.

Descriptores: Motivación; Anciano; Actividad Motora; Ejercicio.

\section{INTRODUÇÃO}

Atualmente, o Brasil encontra-se em uma importante transição sociodemográfica, com estimativa de que a população acima de 65 anos aumente de $2 \%$ a $4 \%$ ao ano nos próximos $40 \operatorname{anos}^{(1)}$. $\mathrm{O}$ aumento da expectativa de vida está geralmente associado ao surgimento de doenças crônicas comuns ao processo de envelhecimento, como processos degenerativos, disfunções musculoesqueléticas, doenças cerebrovasculares e cardíacas, podendo também estar atrelado ao aumento dos custos assistenciais de saúde e ao maior risco de institucionalização e dependência do idoso $^{(2)}$.

O envelhecimento promove diversas adaptações fisiológicas que contribuem para o declínio funcional dos sistemas cardiovascular, musculoesquelético e nervoso central. Tais modificações podem contribuir para a redução nas capacidades física, cognitiva e funcional do idoso, gerando um importante impacto na qualidade de vida $\left(\mathrm{QV}^{(3,4)}\right.$. A literatura coloca que a prática de atividade física visa o fortalecimento muscular, a melhora da flexibilidade e do condicionamento aeróbico, oferecendo benefícios à autonomia funcional em indivíduos com mais de $60 \operatorname{anos}^{(5)}$.

Nesse contexto, o Método Pilates (MP) é uma forma de exercício que vem crescendo em popularidade e ganhando espaço tanto no âmbito do esporte como no da reabilitação, preferencialmente na população de idosos. O MP foi desenvolvido pelo alemão Joseph Hubertus Pilates (1880-1967) durante a Primeira Guerra Mundial, mas somente na década de 1980 passou a ser reconhecido internacionalmente, e nos anos 1990 ganhou popularidade principalmente no campo da reabilitação ${ }^{(6,7)}$.

Devido às necessidades encontradas no processo de envelhecimento e uma grande adesão ao MP, esta pesquisa está apoiada sobre a Teoria da Autodeterminação (TAD) para verificar os fatores motivacionais ${ }^{(8)}$. Ela é muito utilizada em diversas áreas de estudo, incluindo o esporte e a atividade física ${ }^{(6)}$, e explica os diferentes fatores/dimensões motivacionais que levam o indivíduo a aderir, manter ou abandonar a prática da atividade física ${ }^{(9)}$.

Segundo essa teoria, a motivação pode variar de três formas: motivação intrínseca, motivação extrínseca ou amotivação ${ }^{(9)}$. Intrinsecamente motivado é aquele indivíduo que adere à atividade física pela sua própria vontade; a motivação extrínseca ocorre quando a atividade física é realizada com outro objetivo, não partindo do próprio indivíduo; e o indivíduo que não vê a atividade física como algo satisfatório, capaz de trazer algum tipo de benefício, enquadra-se no conceito de amotivação ${ }^{(9,10)}$.

As concepções apresentadas convergem com o conceito de promoção do envelhecimento ativo propagado pela Organização Mundial de Saúde, caracterizado pela experiência positiva de longevidade, com preservação de capacidades e do potencial de desenvolvimento do indivíduo, para que a garantia de condições de vida e de políticas sociais seja uma prerrogativa ${ }^{(11)}$. No âmbito nacional, a Política Nacional da Pessoa Idosa reforça a importância do envelhecimento ativo e saudável, priorizando ações motivadoras e facilitadoras para a adesão de um estilo de vida saudável, considerando a prática regular de atividade física em todos os níveis de atenção ${ }^{(12)}$.

Alguns estudos referentes à motivação e prática de atividade física regular podem ser apontados na literatura, porém, nenhum até então foi relacionado ao $\mathrm{MP}^{(13-17)}$. Além disso, considerando a demanda da população idosa que pratica o $\mathrm{MP}^{(18)}$ e a importância da identificação dos fatores motivacionais para o envelhecimento ativo e saudável ${ }^{(19,20)}$, justifica-se a condução do presente estudo. Posto isso, o objetivo da presente pesquisa foi avaliar os fatores motivacionais de idosos praticantes do Método Pilates e sua relação com o sexo.

\section{MÉTODOS}

Trata-se de uma pesquisa quantitativa, com delineamento transversal e amostra não probabilística por conveniência, constituída de 39 idosos, independentemente do sexo, praticantes do MP. Adotou-se como critério 
de inclusão: praticar o MP há pelo menos três meses consecutivos e ter idade igual ou maior que 60 anos. Os indivíduos que por qualquer motivo não estavam aptos a preencher o questionário de avaliação foram excluídos do estudo.

Todos os estabelecimentos de pilates, incluindo estúdios, academias e clínicas, situados em um bairro da zona norte do Rio de Janeiro-RJ foram convidados a participar do estudo realizado entre os meses de abril e maio de 2014. Recrutaram-se os idosos em nove locais que aceitaram participar do estudo e permitiram a aplicação dos questionários. Realizou-se apenas uma visita a cada um desses estabelecimentos para convidá-los a participar do estudo.

Todos aqueles que aceitaram voluntariamente assinaram o Termo de Consentimento Livre e Esclarecido e posteriormente responderam ao "Inventário de Motivação à Prática Regular de Atividades Físicas (IMPRAF-54)"(21). Esse questionário é composto por 54 itens em nove blocos, subdivididos em seis domínios ("Controle de estresse", "Saúde", "Sociabilidade", "Competitividade", "Estética" e "Prazer"), avaliados por meio de uma escala Likert, com escores específicos para cada domínio, em que o valor mais baixo corresponde à resposta "isto me motiva pouquíssimo", e o valor mais alto significa "isto me motiva muitíssimo". Esse instrumento foi desenvolvido no Brasil e permite avaliar e classificar indivíduos de 13 a 83 anos $^{(21)}$.

A normalidade da distribuição das variáveis foi avaliada pelo teste Shapiro Wilk; e a homocedasticidade, pelo critério de Bartlett. Constatada a distribuição normal das variáveis e homocedasticidade, realizou-se a análise descritiva dos dados com média e desvio padrão, valor mínimo e máximo e percentis oriundos dos escores brutos de cada domínio do instrumento, por sexo. Tabelas normativas são disponibilizadas no manual do instrumento, permitindo comparar o desempenho do indivíduo avaliado com o esperado para cada categoria de classificação de motivação, de acordo com faixa etária e sexo ${ }^{(21)}$. O percentil 60 foi considerado o ponto de corte para classificação dos indivíduos da faixa etária do presente estudo, assim analisados em duas categorias: Percentil $\geq 60$ : "motivação alta" e Percentil<60: "motivação baixa"(10). Os dados formam tabelados e processados no software SPSS 17.0.

Esta pesquisa foi aprovada pelo Comitê de Ética em Pesquisa da Universidade Castelo Branco, Rio de JaneiroRJ, conforme resolução 466/12 do Conselho Nacional de Saúde, processo número 2012/004.

\section{RESULTADOS}

A idade média dos 39 indivíduos avaliados foi de 70 $( \pm 7,8)$ anos, sendo $29(74,3 \%)$ mulheres e $10(25,7 \%)$ homens. Tratando-se dos escores brutos dos seis domínios relacionados à motivação para a prática de atividade física dos participantes, entre o sexo feminino, "Competitividade" $(9,8 \pm 2,8)$ e "Saúde" $(34,3 \pm 4,7)$ obtiveram a menor e a maior média de escore bruto, respectivamente, enquanto para aqueles do sexo masculino, "Estética" $(15,6 \pm 8,7)$ foi o domínio com a média mais baixa e "Competitividade" $(80,3 \pm 26,6)$ foi a média mais elevada. Também foi verificado que as mulheres apresentaram médias maiores do que as dos homens em todos os aspectos, exceto para "Competitividade" e "Sociabilidade" (Tabela I).

Analisando os valores dos percentis baseados nos pontos de corte da tabela normativa do instrumento, "Sociabilidade" obteve o percentil mais alto entre as mulheres (percentil 48), mas todos os domínios analisados foram classificados com "motivação baixa". Para o sexo masculino, somente para o domínio "Sociabilidade" (percentil 70) a classificação "motivação alta" foi obtida (Tabela II).

Tabela I - Média e desvio padrão mínimo e máximo do escore de avaliação de motivação dos idosos para a prática do Método Pilates (IMPRAF - 54). Rio de Janeiro-RJ, 2014.

\begin{tabular}{lccccccc}
\hline & \multicolumn{3}{c}{ Mulheres $(\mathbf{n}=\mathbf{2 9})$} & & \multicolumn{3}{c}{ Homens $(\mathbf{n}=\mathbf{1 0})$} \\
\cline { 2 - 3 } \cline { 6 - 8 } Domínio & Média $( \pm \mathbf{D P})$ & Mínimo & Máximo & & Média $( \pm \mathbf{D P})$ & Mínimo & Máximo \\
\hline Controle de Estresse & $24,1( \pm 8,9)$ & 8 & 38 & & $16,3( \pm 7,7)$ & 8 & 32 \\
Saúde & $34,3( \pm 4,7)$ & 22 & 40 & & $30,3( \pm 5,2)$ & 24 & 40 \\
Sociabilidade & $27,8( \pm 10,2)$ & 8 & 40 & & $28,8( \pm 9,7)$ & 14 & 40 \\
Competitividade & $9,8( \pm 2,8)$ & 8 & 16 & & $80,3( \pm 26,6)$ & 38 & 120 \\
Estética & $21,2( \pm 8,6)$ & 8 & 40 & & $15,6( \pm 8,7)$ & 8 & 32 \\
Prazer & $30,8( \pm 7,5)$ & 10 & 40 & & $29,3( \pm 8,3)$ & 8 & 38 \\
\hline
\end{tabular}

DP: desvio padrão. 
Tabela II - Classificação por percentis para domínios de motivação dos idosos para prática do Método Pilates, segundo tabelas normativas do IMPRAF - 54, por sexo. Rio de Janeiro-RJ, 2014.

\begin{tabular}{lcc}
\hline Domínios & Mulheres $(\mathbf{n}=\mathbf{2 9})$ & Homens $(\mathbf{n}=\mathbf{1 0})$ \\
\hline Controle de Estresse & 36 & 31 \\
Saúde & 47 & 37 \\
Sociabilidade & 48 & $70^{*}$ \\
Competitividade & 24 & 12 \\
Estética & 29 & 18 \\
Prazer & 44 & 47 \\
\hline
\end{tabular}

* classificado como "motivação alta"

\section{DISCUSSÃO}

Buscando descrever a percepção de domínios de motivação ligados à prática do MP por uma população específica de idosos de um bairro da zona norte do Rio de Janeiro-RJ, o presente estudo suscita a discussão de uma temática ainda não explorada exaustivamente na literatura.

A motivação e as perspectivas em relação à prática de atividade física em idosos é um assunto de suma importância evidenciado na literatura. Autores destacam que alguns idosos ainda acreditam que a atividade física é desnecessária, ou mesmo potencialmente prejudicial. Outros reconhecem os benefícios da atividade física, mas relatam uma série de barreiras à prática. Seis barreiras foram citadas como as principais, e a motivação estava entre elas ${ }^{(22)}$.

No Brasil, um estudo realizado na cidade de Florianópolis-SC, em 2008, com 111 idosos de ambos os sexos verificou que "Saúde" e "Sociabilidade" foram os fatores considerados de "alta motivação"(14). Com relação ao presente estudo, achados similares podem ser verificados: as mulheres apresentaram percentis de "Sociabilidade" e "Saúde" mais elevados, e os homens apresentaram o percentil de "Sociabilidade" como o mais alto. Segundo a Teoria da Autodeterminação, compreendese que tem motivação pela saúde aquela pessoa interessada em adquirir, manter e melhorar a saúde; e sociabilidade evidencia a motivação das pessoas que veem na atividade física uma oportunidade para estar com os amigos ${ }^{(21)}$.

Ao considerar o público idoso do presente estudo, a presença de doenças e a recomendação do médico, identifica-se que a motivação pela saúde tem, inicialmente, uma regulação externa. Por sua vez, a literatura aponta que, com o passar do tempo, o indivíduo pode passar a assumir uma regulação identificada ou integrada com tal fator motivacional, principalmente quando passa a considerá-lo importante e aprecia os resultados de sua participação em tal atividade ${ }^{(23)}$.
Outro estudo, realizado na cidade do Recife-PE com 120 idosos de programas de saúde ligados à rede pública, avaliados por um questionário adaptado sobre motivação para a prática esportiva, observou que fatores relacionados à saúde e ao desempenho físico também foram os fatores motivacionais mais relatados entre os idosos, tanto para adesão como para a permanência na atividade física ${ }^{(24)}$.

Em 2014, um estudo comparou os fatores motivacionais para prática de atividade física entre idosos brasileiros e portugueses, com amostra predominantemente feminina nos dois países, e a saúde foi percebida como uma preocupação comum para ambas as realidades culturais ${ }^{(25)}$. Outro recente estudo, realizado com 150 idosos suecos do sexo masculino, com idade entre 50 e 86 anos, também verificou que o principal motivo para realizar atividade física estaria relacionado à saúde ${ }^{(26)}$. Esse mesmo estudo ${ }^{(26)}$ observou que as razões sociais ficaram entre as menos citadas como fator motivacional para atividade física, contrapondo-se aos achados do presente estudo e de outros que relatam as razões sociais como o fator mais importante ${ }^{(27,28)}$ ou o segundo mais importante ${ }^{(29,30)}$. Deve-se considerar que os homens foram sub-representados na maioria desses estudos, bem como na pesquisa em questão; além disso, fatores culturais devem ser fortemente considerados no âmbito da socialização.

Nesse contexto, nenhum estudo avaliou especificamente a motivação para a prática do MP. Todavia, fatores motivacionais que levam os idosos a praticarem outras modalidades de atividade física parecem ser os mesmos identificados no presente estudo para o MP.

As evidências sobre os benefícios do MP para a aptidão física e bem-estar de idosos são consolidadas na literatura, demonstrando grande destaque principalmente em relação a ganho e melhora de força muscular, caminhadas e performances de marcha, atividades da vida diária, estados de humor e $\mathrm{QV}^{(31)}$. Dessa forma, o MP parece atuar de forma pontual nas principais demandas inerentes ao envelhecimento, tornando-se uma forte ferramenta de prescrição de exercício para idosos e indivíduos que buscam uma prática de atividade física regular diferenciada ${ }^{(32)}$. 
Os achados deste estudo e a identificação dos fatores motivacionais para a prática de atividade física de idosos contribuem na elaboração de ferramentas que atendam às expectativas deste público e auxilie na manutenção em longo prazo desses indivíduos de forma ativa. Além disso, colaboram no planejamento de estratégias de promoção de saúde para idosos, visando um envelhecimento saudável e funcional.

Cabe ressaltar que o presente estudo apresenta limitações. O tamanho da amostra foi um fator limitante com importante influência no poder estatístico. A realização do estudo em uma região específica, com amostra por conveniência, inviabiliza que os resultados obtidos sejam extrapolados para outras populações. Análises comparativas mais elaboradas entre os sexos não foram possíveis de ser realizadas devido ao tamanho amostral, mas parecem ser uma interessante estratégia de análise, considerando-se diferentes fatores motivacionais para homens e mulheres. Outra questão a ser analisada refere-se ao fato de que mesmo o instrumento utilizado tendo sido validado para a população idosa, dificuldades inerentes à idade avançada, como limitações visuais, podem dificultar o autopreenchimento de um questionário.

Estudos futuros, com amostras maiores e delineamentos de pesquisa mais sofisticados, são fundamentais para a obtenção de resultados mais robustos, bem como uma avaliação em longo prazo desses fatores motivacionais junto à prática do MP.

\section{CONCLUSÃO}

Fatores relacionados à saúde, sociabilidade e prazer foram determinantes motivacionais entre os idosos avaliados praticantes do Método Pilates.

\section{REFERÊNCIAS}

1. Instituto Brasileiro de Geografia e Estatística - IBGE. Indicadores conjunturais [acesso em 2014 Maio 1]. Disponível em: http://www.ibge.gov.br/home/ estatistica/pesquisas/indicadores.php.

2. Ehrman JK, De Jong A, Sanderson B, editors. ACSM's resource manual for guidelines for exercise testing and prescription. $6^{\text {th }}$ ed. Philadelphia: Lippincott Williams \& Wilkins; 2010.

3. Kirkwood TBL, Austad SN. Why do we age? Nature. 2000;408(6809):233-8.

4. Kendrick D, Kumar A, Carpenter H, Zijlstra GA, Skelton DA, Cook JR. Exercise for reducing fear of falling in older people living in the community.
Cochrane Database Syst Rev. 2014;11:CD009848.

5. Matsudo SM, Matsudo VKR, Barros Neto TL. Efeitos benéficos da atividade física na aptidão física e saúde mental durante o processo de envelhecimento Rev Bras Ativ Fís Saúde. 2000;5(2):60-76.

6. Silva ACLG, Mannrich G. Pilates na reabilitação: uma revisão sistemática. Fisioter Mov. 2009;22(3):449-55.

7. Latey P. Updating the principles of the Pilates methodPart 2. J Bodyw Mov Ther. 2002;6(2):94-101.

8. Reiss S. Intrinsic and extrinsic motivation. Teach Psychol. 2012;39(2):152-6.

9. Balbinotti MAA, Barbosa MLL, Balbinotti CAA, Saldanha RP. Motivação à pratica regular de atividade física: um estudo exploratório. Estud Psicol. 2011;16(1):99-106.

10. Meurer ST, Benedetti TRB, Mazo GZ. Fatores motivacionais de idosos praticantes de exercícios físicos: um estudo baseado na teoria da autodeterminação. Estud Psicol. 2012; 17(2):299-303.

11. World Health Organization - WHO. Non communicable disease prevention and health promotion, ageing and life course. Active ageing: a policy framework [Internet]. Geneva: WHO; 2002 [acesso em 2014 Jul 4]. Disponível em http://www.who.int/ageing/ publications/active/en/index.html

12. Ministério da Saúde (BR), Secretaria de Atenção à Saúde, Departamento de Ações Programáticas Estratégicas. Política nacional da Saúde da Pessoa idosa [Internet]. Brasília (DF): Ministério da Saúde; 2006 [acesso em 2014 Mar 29]. Disponível em: http:// www.saude.gov.br

13. Balbinotti MAA, Capozzoli CJ. Motivação à prática regular de atividade física: um estudo exploratório com praticantes em academias de ginástica. Rev Bras Educ Fís Esp. 2008;22(1):63-80.

14. Meurer ST, Benedetti TRB, Mazo GZ. Teoria da autodeterminação: compreensão dos fatores motivacionais e autoestima de idosos praticantes de exercícios físicos. Rev Bras Ativ Fís Saúde. 2011;16(1):18-24.

15. Aaltonen S, Leskinen T, Morris T, Alen M, Kaprio J, Liukkonen J, et al. Motives for and barriers to physical activity in twin pairs discordant for leisure time physical activity for 30 years. Int J Sports Med. 2012;33(2):15763.

16. Costello E, Kafchinski M, Vrazel J, Sullivan P. Motivators, barriers, and beliefs regarding physical 
activity in an older adult population. J Geriatr Phys Ther. 2011;34(3):138-147.

17. Moschny A, Platen P, Klaassen-Mielke R, Trampisch $\mathrm{U}$, Hinrichs T. Barriers to physical activity in older adults in Germany: a cross-sectional study. Int J Behav Nutr Phys Act. 2011;8:121.

18. Rocha SA, Hartmann C. Pilates: recurso fisioterapêutico como melhoria da qualidade de vida em idosos. FIEP Bulletin On-line [periódico na Internet]. 2012 [acesso em 2014 Maio 1]; 82(1). Disponível em: http:// www.fiepbulletin.net/index.php/fiepbulletin/article/ view/2512

19. Phillips EM, Schneider JC, Mercer GR. Motivating elders to initiate and maintain exercise. Arch Phys Med Rehabil 2004;85(7 Suppl 3):S52-7;quiz S58.

20. Schutzer KA, Graves BS. Barriers and motivations to exercise in older adults. Prev Med 2004;39(5):105661.

21. Barbosa MLL, Balbinotti, AAM. Inventário de Motivação à Prática Regular de Atividade Física (IMPRAF - 54). Laboratório de Psicologia do Esporte - Universidade Federal do Rio Grande do Sul: Porto Alegre, 2006.

22. Franco MR, Tong A, Howard K, Sherrington C, Ferreira PH, Pinto RZ \& Ferreira ML. Older people's perspectives on participation in physical activity: a systematic review and thematic synthesis of qualitative literature. Br J Sports Med. 2015; 0:1-9.

23. Teixeira PJ, Carraça EV, Markland D, Silva MN, Ryan RM. Exercise, physical activity, and self-determination theory: a systematic review. Int J Behav Nutr Phys Act. 2012;9:78.

24. Freitas CMSM, Santiago MDS, Viana AT, Leão AC, Freyre C. Aspectos motivacionais que influenciam a adesão e manutenção de idosos a programas de exercícios físicos. Rev Bras Cineantropom Desempenho Hum. 2007;9(1):92-100.

25. Cavalli AS, Pogorzelski LDV, Domingues MR, Afonso MDR, Ribeiro JAB, Cavalli MO. Motivation of elderly people to engage in physical exercising: a comparative study between two university-based programsBrazil and Portugal. Rev Bras Geriatr Gerontol. 2014;17(2):255-64.
26. Sjörs C, Bonn SE, Lagerros YT, Sjölander A \& Bälter $\mathrm{K}$. Perceived Reasons, Incentives, and Barriers to Physical Activity in Swedish Elderly Men. Interact J Med Res. 2014;3(4):e15.

27. Horne M, Tierney S. What are the barriers and facilitators to exercise and physical activity uptake and adherence among South Asian older adults: a systematic review of qualitative studies. Prev Med. 2012;55(4):276-84.

28. Salehi L, Eftekhar H, Mohammad K, Taghdisi MH, Shojaeizadeh D. Physical activity among a sample of Iranians aged over 60 years: an application of the transtheoretical model. Arch Iran Med 2010;13(6):52836.

29. Päivi M, Mirja H, Terttu P. Changes in physical activity involvement and attitude to physical activity in a 16year follow-up study among the elderly. J Aging Res 2010;174290.

30. Costello E, Kafchinski M, Vrazel J, Sullivan P. Motivators, barriers, and beliefs regarding physical activity in an older adult population. J Geriatr Phys Ther. 2011;34(3):138-47.

31. Bullo V, Bergamin M, Gobbo S, Sieverdes JC, Zaccaria $M$, Neunhaeuserer D, et al. The effects of Pilates exercise training on physical fitness and wellbeing in the elderly: a systematic review for future exercise prescription. Prev Med. 2015;75:1-11.

32. Cruz-Ferreira A, Fernandes J, Laranjo L, Bernardo LM, Silva A. A systematic review of the effects of pilates method of exercise in healthy people. Arch Phys Med Rehab. 2011;92(12):2071-81.

\section{Endereço para correspondência:}

Ricardo Dantas

Av. de Santa Cruz, 1631

Bairro: Realengo

CEP: 21710-255 - Rio de Janeiro - RJ - Brasil

E-mail: ricardodog@gmail.com 